differences, in a study at Sydney Children's Hospital, Australia. Compared to MTLE, seizures in FLE were significantly shorter, more frequent, and occurred predominantly in sleep; EEG abnormalities were often bilateral and of significantly higher frequency; MRI mean frontal cortical volume in FLE was significantly lower than MTLE and controls; and the outcome following surgery was poor. Few were considered optimal surgical candidates because of signs of bilateral disease. Frontal lesions occurred in only $29 \%$ compared to a $94 \%$ temporal lobe lesion rate in patients with MTLE. Etiology was undetermined in the majority of FLE cases. (Lawson JA, Cook MJ, Vogrin S, et al. Clinical, EEG, and quantitative MRI differences in pediatric frontal and temporal lobe epilepsy. Neurology March (1 of 2) 2002;58:723-729). (Reprints: Dr AME Bye, Department of Neurology, Sydney Children's Hospital, High Street, Randwick 2031, Australia).

COMMENT. Frontal lobe epilepsy accounts for more than $20 \%$ of pediatric partial epilepsies in this center. The seizures are of briefer duration, more frequent, and usually nocturnal, compared to temporal lobe epilepsy. Rates of epigastric aura, oral and gestural automatisms, and contralateral limb dystonia are higher, and motor manifestations such as asymmetric tonic, focal clonic, and motor agitation are more prominent. Interictal and ictal EEG abnormalities are not clearly lateralized, and more often synchronous bilateral or independent right or left. MRI detection of a localized lesion is uncommon, and etiology is usually undetermined, although unilateral frontal hypometabolism may be demonstrated by PET and SPECT. Compared to TLE cases, results of surgery for FLE are disappointing.

\title{
INCIDENCE OF ABNORMAL FDG-PET IN PARTIAL EPILEPSY
}

Forty children were studied with EEG, MRI, and F-fluorodeoxyglucose (FDG)PET, within one year of their third unprovoked partial seizure, at the Children's National Medical Center, George Washington University School of Medicine, Washington, DC. The mean age at seizure onset was 5.8 years, mean epilepsy duration 1.1 years, and mean number of seizures 30 (range 3 to 200). An absolute asymmetry index (AI) greater than 0.15 was considered abnormal. Seizure foci were temporal lobe in 33, frontotemporal in 5 , and frontal in 2 . Mean AI for all regions was not different from that obtained in 10 normal young adults. Focal hypometabolism occurred in $8(20 \%)$, all restricted to the temporal lobe, and ipsilateral to the presumed ictal focus. Cerebral metabolic dysfunction may be more related to persistent epilepsy and is uncommon at onset of a partial seizure disorder. (Gaillard WD, Kopylev L, Weinstein S, et al. Low incidence of abnormal FDG-PET in children with new-onset partial epilepsy. A prospective study. Neurology March (1 of 2) 2002;58:717-722). (Reprints: Dr William Davis Gaillard, Department of Neurology, Children's National Medical Center, 111 Michigan Avenue NW, Washington DC, 20010).

COMMENT. Only $20 \%$ of children with recent-onset partial epilepsy have PET evidence of temporal hypometabolism. In adults with refractory TLE, the frequency of temporal lobe hypometabolism is 80 to $85 \%$. A follow-up of the patient population is planned by the authors to determine long-term outcome and possible development of metabolic dysfunction with recurrence of seizures.

In a previous study, the authors had examined the effect of valproate on cerebral metabolism by use of PET. (Gaillard WD et al. Epilepsia 1996;37:515-521). VPA reduced regional cerebral blood flow but not cerebral metabolic rate for glucose in the thalamus. The effect was associated with control of generalized seizures. ( see Progress in Pediatric Neurology III, PNB Publishers 1997;pp40-41). 